\title{
How Exposure to Markets Can Favor Inequity-Averse Preferences ${ }^{* \dagger}$
}

\author{
Robertas Zubrickas ${ }^{\ddagger}$
}

October 28, 2008

\begin{abstract}
Outside the group selection framework, this paper shows how in the presence of general equilibrium effects non-individualistic preferences can be individual fitness maximizing. We present the argument in the model, where individuals share among themselves an endowment and use the proceeds either for immediate consumption or for purchase of consumption goods from merchants on the outside market. Assuming that increased consumption means increased individual fitness, inequity-averse behavior with respect to endowment distribution can be an optimal response to merchants' price discrimination and, eventually, it can lead to the evolution of inequity-averse preferences. One of the model's predictions that the members of a society exposed to external markets behave more pro-socially than do the members of an isolated society is supported by empirical evidence from the related literature.
\end{abstract}

\section{Introduction}

The conventional assumption of economics that people prefer more money to less has been cast into doubt by vast experimental evidence. It has been profusely demonstrated that when making decisions, alongside their own pecuniary interest, people also care, to a larger or smaller extent, about the well-being of other affected parties (for a comprehensive review, see Fehr and Schmidt (2006)). Furthermore, people's behavior, as

*An earlier version of this paper appeared with the title "An Evolutionary Argument for InequityAverse Preferences."

${ }^{\dagger}$ I am indebted to Karl Wärneryd for valuable comments throughout the progress of this paper. I also thank Kaushik Basu, Martin Dufwenberg, and Cheng-Zhong Qin. The financial support from the Jan Wallander and Tom Hedelius Foundation and Inga och Sixten Holmquists stipendiestiftelse is gratefully acknowledged.

${ }^{\ddagger}$ Department of Economics, Stockholm School of Economics, Box 6501, SE-11383 Stockholm, Sweden. E-mail: robertas.zubrickas@hhs.se 
documented in numerous experiments, seems to exhibit certain regular patterns such as, among other things, inequity aversion - which is going to be the main object of this paper - described as "people are willing to give up some material payoff to move in the direction of more equitable outcomes." ${ }^{1}$ Therefore, as an alternative to the conventional assumption, inequity-averse preferences have been proposed, which are, in crude terms, an extension of own-regarding preferences to include inequity-aversion terms (see, e.g., Fehr and Schmidt (1999); Bolton and Ockenfels (2000); Charness and Rabin (2002)).

Further research has also showed that the form of revealed preferences (supposedly, the degree of elicited inequity aversion as well) differ from society to society suggesting that preferences can be context dependent and shaped by the environment people live in (for empirical evidence, see Buchan et al. (2002); Henrich et al. (2001); Henrich et al. (2004); whereas Bowles (1998) offers a systematic review of related theoretical and empirical literature). The most compelling evidence on between-group differences in people's behavior comes from a large scale project conducted in 15 remote primitive societies scattered around the globe, which is documented in the studies Henrich et al. (2001) and Henrich et al. (2004). In particular, with the help of experiments, the researchers have discovered certain regularities in people's revealed amount of sociality, one of which is that the members of a market-integrated society (as measured, primarily, by a society's exposure to external markets) behave on average more pro-socially than do the members of a more isolated society. However, in Henrich et al. (2004, p.50-51) they leave the question what explains these regularities open questing for more research on this important finding, and this is where the current paper attempts to contribute.

This paper offers an evolutionary argument for inequity-averse preferences and shows how specific situational factors can influence their appearance. Specifically, we provide a general-equilibrium framework, where people's preferences for money distribution are endogenously determined by the specified economic factors (such as, for example, exposure to external markets). Subsequently, we demonstrate that inequity aversion can be individual fitness maximizing and eventually be favored by natural or cultural selection. The crux of the presented argument is that in this paper we measure evolutionary fitness not in terms of monetary payoffs but rather in terms of the consumption that monetary payoffs can afford. Since these different measures of fitness in a general equilibrium need not to be equivalent, stark differences in the results can be obtained depending which measure is used. ${ }^{2}$

Specifically, we present the argument in a model — an extended Dictator Game with

\footnotetext{
${ }^{1}$ Fehr and Schmidt (1999, p. 819)

${ }^{2}$ In a similar fashion, Huck and Oechssler (1999) develop an evolutionary argument for revengeful behavior presuming that the individual subjective payoff and subsequent evolutionary fitness resulted from employed strategies are not equivalent. The general models of evolution of preferences (see Ely and Yilankaya (2001); Ok and Vega-Redondo (2001); Dekel et al. (2007)) also differentiate between people's subjective and objective preferences.
} 
consumption - where in equilibrium we obtain that the dictator can be better off (in terms of expected own consumption) by sharing the monetary endowment with others. As a simple example, illustrating the main idea of the paper, consider a two-player Dictator Game with consumption, where the dictator is randomly chosen from the two identical individuals to divide an exogenously given monetary endowment. While individual endowment shares are private information, suppose that the endowment distribution resulting from a split is public information. Also, let an individual's ultimate utility be measured in terms of the affordable consumption of the only good purchased from a single profit-maximizing producer, who charges the price for a unit of the good, produced at zero cost, that maximizes her profits from following trades. It turns out that it isn't optimal for the dictator to keep all the endowment with himself, for in that case the producer targets only the dictator and leaves him no consumer surplus (or rather no information rent). Instead, the dictator could increase his consumption by giving away to the other individual a portion of the endowment large enough to make the rational producer set the price aimed at both individuals, which would leave the richer one - the dictator - with some consumer surplus. Hence, from a conventional utility function for consumption we can obtain a non-monotonic indirect utility function of money that can be interpreted as having underlying inequity-averse preferences for money distribution, the intuition behind which is that by sharing the endowment one can acquire some information rent and, as a result, increase the purchasing power of the own share.

Hence, in the presence of general equilibrium effects individualistic preferences may not render the highest material payoff, or, in other words, may not be favored by natural or cultural selection, an example of which is presented in this paper. Typically, to show how non-individualistic preferences survive evolutionary pressures has required either some group selection argument in standard evolutionary models (for a review, see Bergstrom (2002)) or certain informational assumptions on the part of players and strategic externalities in "indirect" evolutionary models (for a concrete example, see Bester and Güth (1998); for a more general argument, see, e.g., Dekel et al. (2007)), whereas this paper bypasses all of the above: the result hinges on general equilibrium effects. Therefore, this paper rather falls into the "game of life" paradigm, arguing that people's behavior should be examined in a wider social context (see Binmore $(1994,1998)$; or Güth and Napel (2006) for an example related to inequity-averse preferences). Finally, this paper adopts the "indirect" evolutionary approach (see Güth and Yaari (1992); Ely and Yilankaya (2001)) to show that the obtained equilibrium play of the global game is evolutionary stable; though, the standard approach would render the same results as well.

The paper is organized as follows. Section 2 expands the example given above into a more general model and solves it. Section 3 discusses the obtained results, links them to empirical studies, and offers possible extensions. The last section concludes. 


\section{The Model}

\section{$2.1 \quad$ Framework}

After the land rewards a group of farmers ${ }^{3}$ with a publicly observed harvest surplus, henceforth, the endowment $S$, the farmers share it among themselves, and, if used for own consumption, a share $x \in[0, S]$ renders the material payoff of $U^{0}(x), U_{x}^{0}>0, U_{x x}^{0}<0$. Alternatively, in case the group is exposed to external trades, endowment shares can be used as a means of exchange, i.e., as money, to purchase goods from merchants. It is assumed that even if shares, resulting from an endowment split, are only privately known, the ensued income distribution within the group is public information.

Suppose that merchants can offer only one type of goods - "the good" - which, on the other hand, can be produced of various quality $q$ greater than or equal to some very small $\varepsilon>0$ (this condition is for modeling purposes as later explained) with the production function $C(q), C_{q}>0, C_{q q}>0$, and the returns to scale from producing a given variety are constant. Assuming that any farmer has a demand for at most one unit of the good, and depending on the observed income distribution within the group and on the outside market structure, as more precisely specified below, merchants offer the farmers a menu of price-quality $(p, q)$ bundles of the good to choose from, where the price $p$ is gauged in terms of the endowment. The consumption of a $(p, q)$ unit and of the remainder of the endowment share $x$ renders a farmer the material payoff of $U^{G}(x-p, q), U_{q}^{G}>0, U_{q q}^{G}<0, U_{x}^{G}>0, U_{x x}^{G}<0, U_{q x}^{G}>0$. The farmer will consider purchasing the good only if it results in a non-negative net utility level $U$, defined as $U(q, x, p) \equiv U^{G}(x-p, q)-U^{0}(x)$, which needs to be greater than or equal to 0 for the trade to take place. Correspondingly, the properties of the net utility function $U$ are $U_{x}>0, U_{x x} \leq 0, U_{q}>0, U_{q q} \leq 0$ and $U_{q x}>0 .{ }^{4}$ For convenience, let the function $U$ be of the quasi-linear form in the price $p$ :

$$
U(q, x, p)=V(q, x)-p
$$

Finally, we shall consider three different scenarios of the outside market structure: 1) merchants are absent, i.e., the farmers' economy is autarkic, 2) there is a monopolist profit-maximizing merchant, and 3) the outside market is that of perfect competition,

\footnotetext{
${ }^{3}$ The "farmers" are chosen to make an allusion to the historical labor division into farmers, nomads, and merchants, which could potentially serve as a "real life" example in the subsequent argument about the evolution of inequity-averse preferences for money distribution. In addition, by a "farmer" economy it is referred to the primitive societies in Henrich et al. (2004), from which comes the empirical support of the model's results.

${ }^{4}$ All the listed properties of the utility function $U$ are related to consumer preferences for normal goods (as in, e.g., Mas-Colell et al. (1995)). In particular, the positive partial derivative $U_{x}$ implies that a richer individual derives a higher utility from the consumption of the good (due to, say, smaller opportunity costs). Similarly, the positive cross derivative $U_{q x}$ can be interpreted as that a richer consumer values quality more, which can be motivated by the convexity of the Engel curves for high-quality goods.
} 
i.e., there are many competing merchants.

\subsection{Game and natural selection}

Along the lines of the above framework, consider a large population of farmers randomly and repeatedly matched to form separate groups of two farmers, and every group is endowed with the same-size endowment $S$. In a group, Nature randomly selects a farmer, henceforth, the dictator, to divide the endowment into shares $s \in[0, S]$ for herself and $(S-s)$ for the other farmer. When selected to split the endowment, farmers maximize their subjective preferences over the endowment split $(s, S-s)$ (or, to put it differently, their preferences for money distribution), and these preferences are characterized by the utility function $U^{S}$ with the preference parameter $\bar{s} \in[0, S]$ such that the (subjective) utility accrued to a farmer-dictator with a preference parameter $\bar{s}$ is

$$
U^{S}(s, \bar{s})=-|s-\bar{s}|
$$

Therefore, in what follows, a farmer of preference type $\bar{s}$, when sharing the endowment, will always keep $\bar{s}$ for herself, leaving $S-\bar{s}$ for the other farmer in the match. ${ }^{5}$ Next, suppose that in the population the distribution of subjective preference types is given by some distribution $F$ over $[0, S]$.

However, the objective payoffs from a split, or an evolutionary fitness, are measured by the resulting material payoffs $U^{0}$ and/or $U^{G}$, which, on the other hand, depend both on the own endowment share and on the menu of consumption bundles offered on the outside market. Importantly, knowledge of other players' preferences or the population distribution of preferences won't play any role in this game, which, though, isn't generally the case (see, e.g., Ok and Vega-Redondo (2001) or Dekel et al. (2007)). Next, for modeling convenience, merchants are assumed to design consumption bundles for every match separately, and these bundles aren't available for the farmers from other matches. Furthermore, in what follows, merchants shall behave exclusively in the profit maximizing way, which implies that merchants' market structure and their optimal play will be embedded in the form of the players' (i.e., farmers') material payoff function, allowing us to "prune" the production and consumption stages and to consider the reduced game only.

As already specified above, we distinguish three cases of merchants' market: the autarky, the monopolist market, and the perfectly competitive market, which give rise to three different material payoff functions of the farmers, and we shall analyze these

\footnotetext{
${ }^{5}$ Whereas we are following the "indirect" evolutionary approach (Güth and Yaari (1992) and Ely and Yilankaya (2001)), alternatively, we could allow that farmers split the endowment according to some pre-programmed rule as in standard evolutionary models, see Weibull (1995). Due to the specificity of the studied game, the two approaches would render identical results, which, though, generally is not necessarily the case (e.g., Huck and Oechssler (1999)).
} 
three cases in three different games $\Gamma^{A}, \Gamma^{M}$, and $\Gamma^{C}$, respectively. Each of these games is just a Dictator Game with modified payoffs. In particular, in every game, there are two players (two farmers), their action space is to choose an endowment share $s \in[0, S]$ for himself or herself, and the payoffs to the players from their actions are their expected material payoffs (evolutionary fitness), as will be defined below. Finally, every player has subjective preferences over endowment splits characterized by a parameter $\bar{s}$, according to which he or she divides the endowment. ${ }^{6}$ Then, for every game examined separately, we shall tackle the question which subjective preferences yield greater material payoffs and, accordingly, will be favored by natural selection, with their share increasing in the population at the expense of less successful preferences?

More precisely, we adopt the "indirect" evolutionary approach with a static stability concept of equilibrium that in the equilibrium no mutation can give a higher payoff than that of the incumbent types. Based on the results of Ely and Yilankaya (2001) and applying them to our setting, evolution will select those subjective preferences, or, equivalently, actions over the endowment split, which constitute an equilibrium of the global games $\Gamma^{A}, \Gamma^{M}$, and $\Gamma^{C}$, respectively, as more accurately discussed below; and we shall call those preferences evolutionary stable. ${ }^{7}$

\subsection{Equilibrium play in a match}

\section{Case 1: Autarky}

Consider game $\Gamma^{A}$, where farmers aren't exposed to any external trades, making it just a standard two-player Dictator Game. The material payoff from an endowment share $x$ is just $U^{0}(x)$, so, given the optimal play of the players (with respect to their subjective preferences), the expected material payoff to a farmer of preference parameter $\bar{s}$ when matched with a farmer of preference parameter $\bar{s}^{\prime}$ is

$$
\pi\left(\bar{s}, \bar{s}^{\prime}\right)=0.5 U^{0}(\bar{s})+0.5 U^{0}\left(S-\bar{s}^{\prime}\right)
$$

More generally, in game $\Gamma^{A}$ the payoff (evolutionary fitness) to a preference type $\bar{s}$, given a population distribution of subjective preferences $F$, is the average material payoff $\Pi(\bar{s})$, defined as

$$
\Pi(\bar{s})=0.5 U^{0}(\bar{s})+0.5 E_{\bar{s}^{\prime} \in C(F)} U^{0}\left(S-\bar{s}^{\prime}\right),
$$

\footnotetext{
${ }^{6}$ Referring back to the information structure of the model, we could also think of farmers as living behind the "veil of uncertainty" about external markets, so that they don't have a perfect foresight of what "global game" they are playing, and, therefore, they just divide the endowment according to their subjective preferences only.

${ }^{7}$ Ely and Yilankaya (2001) studies finite games, while in our model the action space is allowed to be infinite: $s \in[0, S]$. However, since we design our games in such a way that the existence of equilibrium is not an issue, then the results of Ely and Yilankaya (2001) apply to our setting as well despite a continuous action space. Alternatively, we could make our studied games finite by simply discretizing the players' action and preference spaces, and then the results of Ely and Yilankaya (2001) would apply directly.
} 
where $C(F)$ is the support of the distribution $F$, and $E$ is the expectations operator.

Since the second term of the above fitness expression doesn't depend on the own preference type, the highest fitness is attained for the type $\bar{s}=S$ because of $U_{x}^{0}>0$. Hence, the equilibrium of $\Gamma^{A}$ is the endowment share $s^{A}=S$, resulting in the endowment split $(S, 0)$, which implies that in such an autarkic setting selfish types would prevail.

\section{Case 2: Monopoly}

In game $\Gamma^{M}$, to specify payoffs to the farmers, first, we need to solve for the optimal consumption bundles offered by the monopolist profit-maximizing merchant. From the merchant's perspective, it is a mechanism design problem with hidden information, for a potential customer's wealth, i.e., his endowment share, is his private information. Once the menu of bundles is set, it is not subject to change, by which we rule out the possibility of updating the merchant's beliefs about prospect buyers' wealth distribution after some trade has taken place (alternatively, we could have assumed that at the last stage only one trade with a random farmer takes place). The exposition of the merchant's problem follows closely Mussa and Rosen (1978).

\section{Merchant's problem}

Given the publicly observed information about the income distribution followed the endowment split in the match, which is the probability distribution for the endowment shares $\tilde{s}_{1}$ and $\tilde{s}_{2}$, with $\tilde{s}_{1} \leq \tilde{s}_{2}$, the monopolist merchant designs an assortment of the good $\left\{\left(p_{j}, q_{j}\right)\right\}_{j=1}^{n}$, aimed at both $(n=2)$ or just the richer farmer $(n=1)$, in order to maximize his expected profit $0.5\left(p_{1}-C\left(q_{1}\right)\right)+0.5\left(p_{2}-C\left(q_{2}\right)\right)$. Since there is no need to state the problem and solve it formally, for the solution to this type of problems is well established in the contract theory literature, see, e.g., the aforementioned Mussa and Rosen (1978), once the specific conditions are met (e.g., in our model the singlecrossing property is ensured by the assumption of the positive cross derivative of net utility function $U, U_{q x}>0$ ). Hence, below we immediately proceed with describing the results, given various cases of the endowment split.

In the special case of the equal endowment split $\tilde{s}_{1}=\tilde{s}_{2}=S / 2$, the merchant offers one price-quality allocation $(p, q)$ such that

$$
\begin{aligned}
& C_{q}(q)=V_{q}\left(q, \tilde{s}_{2}\right), \\
& p=V\left(q, \tilde{s}_{2}\right),
\end{aligned}
$$

which coincides with the first-best allocation in the symmetric information case, where consumers are left with no consumer surplus.

For an uneven split, $\tilde{s}_{1}<\tilde{s}_{2}$, two cases need to be distinguished, when optimally: 1 ) the merchant serves both farmers, and 2) the merchant shuns the poorer farmer and offers a single allocation only to the richer farmer. When the merchant serves both farmers, 
the optimal price-quality bundles $\left(p_{1}, q_{1}\right)$ and $\left(p_{2}, q_{2}\right)$ are given by

$$
\begin{gathered}
C_{q}\left(q_{2}\right)=V_{q}\left(q_{2}, \tilde{s}_{2}\right), \\
C_{q}\left(q_{1}\right)=2 V_{q}\left(q_{1}, \tilde{s}_{1}\right)-V_{q}\left(q_{1}, \tilde{s}_{2}\right),
\end{gathered}
$$

and the prices $p_{1}$ and $p_{2}$ follow from $U\left(q_{1}, \tilde{s}_{1}, p_{1}\right)=0$, which is the binding individual rationality constraint of the poor type, and $U\left(q_{2}, \tilde{s}_{2}, p_{2}\right)=U\left(q_{1}, \tilde{s}_{2}, p_{1}\right)$, which is the binding incentive-compatibility constraint, respectively. In this case, the richer consumer enjoys the information rent of the size $U\left(q_{2}, \tilde{s}_{2}, p_{2}\right)$, while the poorer is left with none. The condition for both farmers being served is the quality $q_{1}$ from (5) being greater than or equal to $\varepsilon$, or $\tilde{s}_{1} \geq s^{*}$, where the threshold value $s^{*}$ is defined as

$$
s^{*}=\left\{\tilde{s}_{1}: \tilde{q}_{1}\left(\tilde{s}_{1}\right)=\varepsilon\right\}
$$

where $\tilde{q}_{1}\left(\tilde{s}_{1}\right)$ is the quality function with respect to $\tilde{s}_{1}$ (whereas $\tilde{s}_{2}$ is replaced with $S-\tilde{s}_{1}$ ) defined from (5), and $\varepsilon>0$ is the smallest quality level that the merchant can feasibly offer. $^{8}$

Hence, in case the endowment split results in $\tilde{s}_{1} \leq s^{*}$, the merchant doesn't serve the poorer farmer, but instead he offers the first-best allocation to the richer farmer as in (2) and (3), but leaving her with no information rent.

\section{Evolutionary fitness}

As in the autarky case, in game $\Gamma^{M}$ the payoffs to players, or their evolutionary fitness, are the expected material payoffs. Given a population distribution of subjective preferences $F$, the evolutionary fitness of preference type $\bar{s}$ is

$$
\Pi(\bar{s})=0.5 Y(\bar{s})+0.5 E_{\bar{s}^{\prime} \in C(F)} Y\left(S-\bar{s}^{\prime}\right)
$$

where $C(F)$ is the support of the distribution $F$, and $E$ is the expectations operator, and the function $Y$ is the indirect utility function, which measures the material payoff from the own endowment share, given the optimal play of the merchant. In particular, the indirect utility function $Y$ of endowment share $\tilde{s}$ (with the other player's share accordingly being $S-\tilde{s})$ is defined as

$$
Y(\tilde{s})=\left\{\begin{array}{ccc}
U^{0}(\tilde{s}) & \text { if } & \tilde{s} \leq S / 2 \\
U^{0}(\tilde{s})+U\left(q_{2}, \tilde{s}, p_{2}\right) & \text { if } & S / 2<\tilde{s} \leq S-s^{*} \\
U^{0}(\tilde{s}) & \text { if } & S-s^{*}<\tilde{s} \leq S
\end{array}\right.
$$

where $\left(p_{2}, q_{2}\right)$ is the price-quality allocation aimed at the richer consumer as defined

\footnotetext{
${ }^{8}$ Had we $q \in[0, \infty)$, then the threshold value, for which still two consumption bundles are offered, would not be precisely determined, as wouldn't be then the equilbrium of game $\Gamma^{M}$.
} 


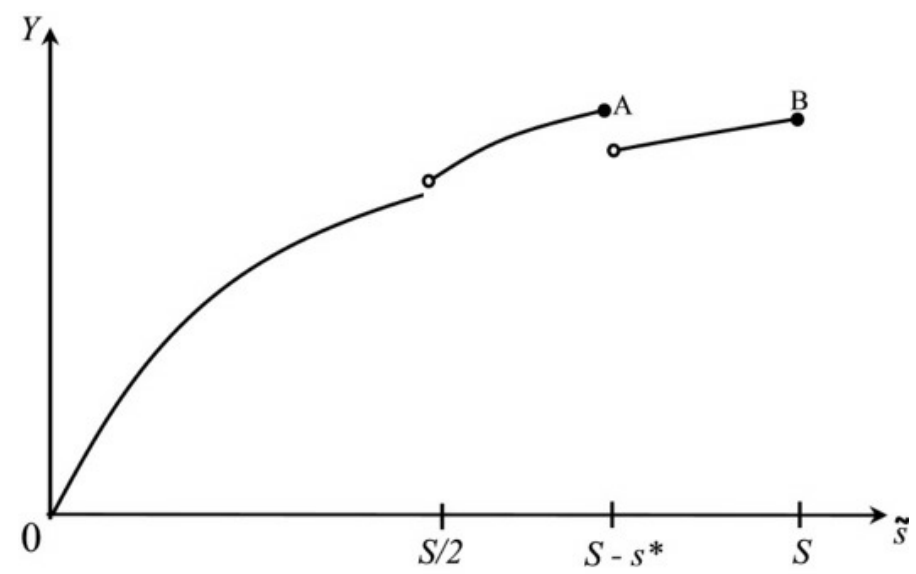

Figure 1. The indirect utility (material payoff) function $Y$

above; and $s^{*}$ is the threshold value as in (6) that provides the condition when both farmers are served.

From the above function's definition, we can observe that for the values of argument $\tilde{s}$ inside the interval $\left(S / 2, S-s^{*}\right]$ the payoff function $Y$ takes a form different from the form it takes when the argument is outside this interval. As discussed above, this is due to information rents available when differences in endowment shares are not too large, otherwise, because of the merchant's full-rent extraction a farmer enjoys only her reservation utility $U^{0}(\tilde{s})$. Specifically, there is an upward shift $U\left(q_{2}, \tilde{s}, p_{2}\right)$-an information rent - in the value of function $Y$ at the interval $\left(S / 2, S-s^{*}\right]$, which results in two discontinuities at the points $\tilde{s}=S / 2$ and $\tilde{s}=S-s^{*}$, see Figure 1. Since the function value drops after the second discontinuity point (see for point $\mathrm{A}$ in the figure), which happens when the merchant shuts down on the poorer consumer and strips the richer one of any information rents, it is not obvious at which point the function $Y$ achieves its global maximum: at $\tilde{s}=S-s^{*}$ (point A) or at $\tilde{s}=S$ (point B). In other words, it is not obvious from the material payoff perspective whether the dictator should keep all the endowment with herself, because doing so leaves her without any information rent, which is otherwise available if she gives away at least $s^{*}$ to the other farmer. It depends on the size of the information rent and the form of the utility functions. Formally, if

$$
U^{0}\left(S-s^{*}\right)+U\left(q_{2}, S-s^{*}, p_{2}\right) \geq U^{0}(S)
$$

or

$$
U^{G}\left(q_{2}, S-s^{*}-p_{2}\right) \geq U^{0}(S)
$$

where $\left(p_{2}, q_{2}\right)$ is the allocation aimed at the richer farmer for the endowment split $(S-$ $\left.s^{*}, s^{*}\right)$, then the dictator attains the highest material payoff when she shares the endowment with the other farmer giving the latter the share $s^{*}{ }^{9}$ Intuitively, it is to require

\footnotetext{
${ }^{9}$ We don't formally show that or provide a condition when the function $Y$ attains its maximum at
} 
that farmers after a certain point get quickly satiated with the consumption of their own endowment shares and value the outside good highly enough.

Therefore, returning to evolutionary fitness expression (7), we can see that in case condition (9) holds the highest expected material payoff is accrued to the farmers of preference type $\bar{s}=S-s^{*}$, and, correspondingly, the equilibrium split in game $\Gamma^{M}$ is $\left(S-s^{*}, s^{*}\right)$. In other words, when the farmers are exposed to external trades run by monopolist merchants, the inequity-averse preferences may get eventually favored by natural selection, which is to prevent the full-rent extraction by merchants.

\section{Case 3: Perfect competition}

Consider game $\Gamma^{C}$, where there are many competing merchants on the outside market. Given that all merchants' profits have to be equal to 0 in perfect competition, the competitive solution to a merchant's problem is easily described. The offered quality level has to be as in the first-best case, while the price has to be equal to the total cost of producing that particular quality. Therefore, the price-quality allocation $\left(p_{j}, q_{j}\right)$ aimed at an income level $\tilde{s}_{j}$ is determined by (2) for the quality $q_{j}$, and the price $p_{j}=C\left(q_{j}\right)$, which, unlike in the monopoly case before, is not a function of consumer preference parameter $\left(\tilde{s}_{j}\right.$ in our case). Following the same logic as before, it is easy to see that selfish farmers with $\bar{s}=S$ will attain the highest material payoff, and this type of preferences should survive evolutionary pressures.

\section{Discussion}

The following proposition summarizes the resultant evolutionary stable preferences for the analyzed environments.

Proposition 1 In the two-player Dictator Game with external trades as studied above, the evolutionary stable preference types $\bar{s}_{\text {es }}$ with respect to the endowment $S$ split are

- with no external trade $-\bar{s}_{\text {es }}=S$;

- with the monopolist merchant - if condition (9) holds, then $\bar{s}_{e s}=S-s^{*}$, where $s^{*}$ is defined as in (6); otherwise, $\bar{s}_{e s}=S$;

- with a competitive merchant $-\bar{s}_{\text {es }}=S$.

The main result of this proposition is that external factors, such as market structure, can have influence on people's behavior and the shape of their preferences. In particular, material inequity aversion in money sharing decisions could have evolved as a response to

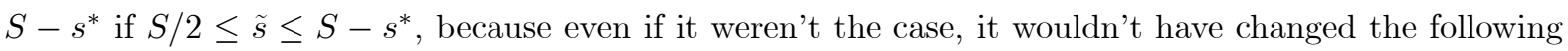
argument, as long as condition (9) holds for some $\tilde{s} \in(S / 2, S]$. 
some monopolistic powers on the markets. The obtained results can also be interpreted as even if people are intrinsically selfish (with regard to own consumption), they can still exhibit behavior as if they have inequity-averse preferences (for money distribution). Referring to the evolutionary literature of the standard "direct" approach, the model argues that in the presence of some general-equilibrium externalities individual selection can also favor the evolution of other-regarding preferences, showing which has typically required some form of group selection (see, e.g., Bergstrom (2002)).

Needless to say that within the studied framework with different levels of competition or monopoly on the outside market other forms of evolutionary stable preferences could emerge. The overall prediction of a more general model would be that the more monopolist markets are, the less selfish people should become.

\subsection{Endogeneity of inequity aversion}

The model shows how people's preferences for money distribution can possibly be endogenous to the economic environment they live in. Given that in real life merchandise markets typically possess some degree of monopoly, the main prediction of the model is that the members of more isolated societies are less likely to behave pro-socially than do the members of societies, exposed to external markets. Most importantly, this prediction is consistent with empirical observations on people's revealed preferences for money distribution, obtained in laboratory experiments run in various countries.

It has been demonstrated that people's preferences underlying their exhibited behavior in money-sharing decisions are not uniform around the world and are rather shaped by socioeconomic factors (see, e.g., Henrich et al. (2001); Henrich et al. (2004); Buchan et al. (2002)). As already noted in the introduction, the most compelling evidence in support of our theoretical findings comes from the empirical project run in 15 primitive societies, which is documented in Henrich et al. (2001) and Henrich et al. (2004). The aim of this project was to look into the foundations of human sociality and its origins, and studying primitive societies could possibly shed light on the evolution of people's behavior in modern civilized societies (where people's behavior is less diverse, see Roth et al. (1991)). The researchers found that people's behavior as revealed in playing ultimatum, public good, and dictator games differ across different groups, and that there are certain regularities in the documented differences. One of the regularities relevant to our model is that the members of a more isolated society behave less pro-socially than do those living in more market-integrated societies (as measured by, among other things, exposure to external markets). This empirical finding is in line with our theoretical predictions

suggesting that our developed model could be seen as a theoretical explanation why people behave more pro-socially when their economic activity goes beyond their habitat. 


\subsection{Model extensions and research directions}

In practice, within a given society the distribution of people's preferences is more diverse than just one type of preferences (see, e.g., Fehr and Schmidt (1999)). For our model to achieve a non-trivial distribution of evolutionary stable preferences, we could introduce some randomness in the structure of the outside market, say, by making it subject to random changes from more competition to less competition and vice versa. Then, by putting the farmers behind a "veil of uncertainty" about the structure of the market, we could obtain more types than one to survive evolutionary forces, possibly getting the model's theoretical patterns of preference distribution closer to empirical ones. Alternatively, we could elaborate the above model by introducing a noisy signal that merchants receive about the income distribution resulted from an endowment split, which would also allow us to obtain a more diverse set of evolutionary stable preferences.

In a similar fashion, we can think of other mechanisms affecting the form of revealed (inequity-averse) preferences. For instance, within our model, consider the effect on people's optimal behavior after the introduction of a uniform sales tax on the outside good. If the public authority aims at maximizing tax revenues, then the model would predict people responding to the tax by reducing inequality in wealth on the grounds similar to the case with a monopolist merchant. On the other hand, if the public authority's imposed tax is negligible, then it wouldn't have any impact on people's behavior. In other words, the importance of the government's role in the economy can also shape the appearance of people's preferences, with more central role adding to inequity aversion among people.

An interesting extension would also be to consider an $N$-player Dictator Game, where the number of farmers, matched to play the Dictator Game, is larger than 2 (similarly to the framework in Ok and Vega-Redondo (2001)). Qualitatively, it shouldn't change the results: in certain cases, inequity-averse preferences should still render the highest material payoff. Interestingly, however, in game $\Gamma^{M}$ it may not be optimal (from the material payoff perspective) for the dictator to split the remaining endowment evenly among the rest of the players provided he finds optimal to give away some of the endowment. Instead, the dictator can do better by dividing the remaining endowment unevenly as it can be seen from the special case of $N=3$ and $V(q, x)=q x$, which at the same time poses an interesting question what is the optimal income distribution from the dictator's perspective in the game with more than two players.

\section{Concluding remarks}

We have argued that the inequity-averse preferences of the form as documented in laboratory experiments may be a product of natural or cultural selection. Inequity aversion 
to money distribution can be acquired as a response to the existing socioeconomic environment, an example of which has been shown in the text, suggesting the endogeneity of revealed preferences.

The presented findings can be thought as an attempt to reconcile experimental evidence with the conventional economic theory; though, the question what people's preferences should be used in economic modeling remains still open. In a partial equilibrium analysis, using the form of preferences that are most characteristic of the society may probably render more accurate predictions than using the conventional assumption of own-regarding preferences. However, if a modeled policy change may have a substantial general-equilibrium reach, then, along the lines of the presented model, it may also affect people's preferences through the social transmission of behavioral traits, complicating predictions of the modeled change in a longer run.

Surely, more empirical research needs to be done exploring the interdependence between the environment and preferences. Regarding the findings of this paper, the link between people's preferences for money distribution and market structure, as conjectured above, could be empirically more closely examined than it was done in Henrich et al. (2004), for necessary empirical data can be obtained with the causation running from markets to preferences, which is left for future research.

\section{References}

Bergstrom, T. C. (2002). Evolution of social behavior: Individual and group selection. Journal of Economic Perspectives 16(2), 67-88.

Bester, H. and W. Güth (1998). Is altruism evolutionary stable? Journal of Economic Behavior \& Organization 34(2), 193-209.

Binmore, K. (1994). Game Theory and the Social Contract - Volume I: Playing Fair. MIT Press.

Binmore, K. (1998). Game Theory and the Social Contract - Volume II: Just Playing. MIT Press.

Bolton, G. E. and A. Ockenfels (2000). Erc: A theory of equity, reciprocity, and competition. American Economic Review 90(1), 166-193.

Bowles, S. (1998). Endogenous preferences: The cultural consequences of markets and other economic institutions. Journal of Economic Literature 36(1), 75-111.

Buchan, N. R., R. T. A. Croson, and R. M. Dawes (2002). Swift neighbors and persistent strangers: A cross-cultural investigation of trust and reciprocity in social exchange. American Journal of Sociology 108(1), 168-206. 
Charness, G. and M. Rabin (2002). Understanding social preferences with simple tests. Quarterly Journal of Economics 117(3), 817-869.

Dekel, E., J. C. Ely, and O. Yilankaya (2007). Evolution of preferences. Review of Economic Studies 74(3), 685-704.

Ely, J. C. and O. Yilankaya (2001). Nash equilibrium and the evolution of preferences. Journal of Economic Theory 97(2), 255-272.

Fehr, E. and K. M. Schmidt (1999). A theory of fairness, competition, and cooperation. Quarterly Journal of Economics 114(3), 817-868.

Fehr, E. and K. M. Schmidt (2006). The economics of fairness, reciprocity and altruismexperimental evidence and new theories. In S.-C. Kolm and J. M. Ythier (Eds.), Handbook on the Economics of Giving, Reciprocity and Altruism, Volume 1, pp. 615691. Elsevier.

Güth, W. and S. Napel (2006). Inequality aversion in a variety of games - an indirect evolutionary analysis. Economic Journal 116(514), 1037-1056.

Güth, W. and M. Yaari (1992). An evolutionary approach to explain reciprocal behavior in a simple strategic game. In U. Witt (Ed.), Explaining Process and ChangeApproaches to Evolutionary Economics, pp. 23-34. The University of Michigan Press, Ann Arbor.

Henrich, J., R. Boyd, S. Bowles, C. Camerer, E. Fehr, and H. Gintis (Eds.) (2004). Foundations of Human Sociality: Economic Experiments and Ethnographic Evidence from Fifteen Small-Scale Societies. Oxford University Press.

Henrich, J., R. Boyd, S. Bowles, C. Camerer, E. Fehr, H. Gintis, and R. McElreath (2001). In search of homo economicus: Behavioral experiments in 15 small-scale societies. American Economic Review 91(2), 73-78.

Huck, S. and J. Oechssler (1999). The indirect evolutionary approach to explaining fair allocations. Games and Economic Behavior 28(1), 13-24.

Mas-Colell, A., M. D. Whinston, and J. R. Green (1995). Microeconomic theory. Oxford University Press.

Mussa, M. and S. Rosen (1978). Monopoly and product quality. Journal of Economic Theory 18(2), 301-317.

Ok, E. A. and F. Vega-Redondo (2001). On the evolution of individualistic preferences: An incomplete information scenario. Journal of Economic Theory 97(2), 231-254. 
Roth, A. E., V. Prasnikar, M. Okuno-Fujiwara, and S. Zamir (1991). Bargaining and market behavior in jerusalem, ljubljana, pittsburgh, and tokyo: An experimental study. American Economic Review 81(5), 1068-1095.

Weibull, J. (1995). Evolutionary Game Theory. MIT Press. 